\title{
Terapia nutricional a pacientes con insuficiencia respiratoria aguda y ventilación mecánica no invasiva: revisión narrativa de la literatura
}

\author{
Nutritional therapy in patients with acute respiratory failure and noninvasive \\ ventilation: narrative literature review \\ Terapia nutricional a pacientes com insuficiência respiratória aguda e ventilação mecânica \\ não invasiva: uma revisão narrativa da literatura
}

\author{
Iván Armando Osuna-Padilla ${ }^{*}$, Patricia Paulina Maldonado-Valadez ${ }^{2}$, Sebastian Rodríguez-Llamazares ${ }^{3}$ \\ Recibido: 7 de mayo de 2019. Aceptado para publicación: 5 de septiembre de 2019. \\ Publicado en línea: 9 de noviembre de 2019 \\ https://doi.org/10.35454/rncm.v3n1.02
}

\section{Resumen}

La instauración de la ventilación mecánica no invasiva impacta de forma negativa el estado nutricional del paciente con insuficiencia respiratoria aguda. El deterioro de la musculatura respiratoria, secundaria a la prescripción de ayuno, afecta la capacidad para sostener niveles adecuados de ventilación.

Objetivo: analizar la literatura científica respecto al abordaje nutricional del paciente con insuficiencia respiratoria aguda y ventilación mecánica no invasiva. Además, proponer un algoritmo de abordaje nutricional para prevenir el deterioro y mejorar el estado nutricional de pacientes con dicha condición clínica.

Metodología: la búsqueda bibliográfica fue realizada en PubMed e Hinari, utilizando las palabras clave (non invasive ventilation $O R$ noninvasive ventilation) AND (nutrition OR nutritional OR intake). Se incluyeron 4 estudios publicados de enero de 2000 a mayo de 2019, realizados en adultos con lesión pulmonar aguda o insuficiencia respiratoria aguda. Se consultaron además las guías y documentos de práctica segura de ESPEN y ASPEN para el desarrollo del algoritmo de abordaje nutricional.

Resultado: en la actualidad, no existe consenso para el abordaje nutricional de esta población. Sin embargo, considerando las asociaciones entre el déficit de energía y proteína, y los desenlaces clínicos reportados en el paciente hospitalizado, resulta fundamental la implementación de protocolos de abordaje nutricional.

\section{Summary}

Background: In acute respiratory failure patients, noninvasive ventilation has a negative impact on nutritional status. Respiratory muscles deterioration secondary to nil per os prescription affects ventilator capacity.

Aim: The aim of this review was to analyze the literature of nutritional therapy in patients with acute respiratory failure and noninvasive ventilation. Additionally, we proposed and algorithm of nutritional intervention.

Method: The search was conducted using PubMed and Hinari. Key terms included (non invasive ventilation OR noninvasive ventilation) AND (nutrition OR nutritional OR intake). Inclusion criteria for this review include primary articles; human adult's studies only and individuals with acute lung injury or acute respiratory failure. Four studies met the inclusion criteria. And additionally review of ASPEN and ESPEN safes practices and guidelines was conducted to propose an algorithm for nutritional intervention.

Result: Currently, there are no consensus documents for the nutritional therapy of this population, however, considering the associations between the deficit of energy and protein and the clinical outcomes reported in the hospitalized patients, is essential the implementation of protocols and promoting optimal nutritional therapy for each patient in order to malnutrition treatment or prevention.

\section{Resumo}

O estabelecimento de ventilação mecânica não invasiva afeta negativamente o estado nutricional do paciente com insuficiência respiratória aguda. A deterioração da musculatura respiratória, secundária à prescrição do jejum, afeta a capacidade de manter níveis adequados de ventilação.

Objetivo: analisar a literatura científica sobre a abordagem nutricional de pacientes com insuficiência respiratória aguda e ventilação mecânica não invasiva. Além disso, propor um algoritmo de abordagem nutricional para evitar a deterioração e melhorar o estado nutricional dos pacientes com a referida condição clínica.

Metodologia: a pesquisa bibliográfica foi realizada em PubMed e Hinari, utilizando as palavras-chave (non invasive ventilation $O R$ noninvasive ventilation) e (nutrition OR nutritional OR intake). Foram incluídos quatro estudos publicados de janeiro de 2000 a maio de 2019 , realizados em adultos com lesão pulmonar aguda ou insuficiência respiratória aguda. Também foram consultados os guias e documentos de práticas seguras da ESPEN e ASPEN, para o desenvolvimento do algoritmo de abordagem nutricional.

Resultado: atualmente, não há consenso para a abordagem nutricional desta população. No entanto, considerando as associações entre o déficit de energia e proteína e os desfechos clínicos relatados no paciente hospitalizado, a implementação de protocolos de abordagem nutricional é essencial. 
Conclusión: el paciente con ventilación mecánica no invasiva presenta un consumo subóptimo de nutrientes. La implementación de algoritmos de abordaje nutricional permitirá prevenir el deterioro y mejorar el estado nutricional de este grupo de pacientes.

Palabras clave: ventilación mecánica no invasiva, terapia nutricional, insuficiencia respiratoria aguda.
Conclusion: Suboptimal nutrients intake is present in patients with noninvasive ventilation. Implementation of a nutritional intervention algorithm is important in order to improve nutritional status in this clinical condition.

Keywords: Noninvasive ventilation; Nutrition therapy; Respiratory insufficiency.
Conclusão: o paciente com ventilação mecânica não invasiva apresenta um consumo subótimo de nutrientes. A implementação de algoritmos de abordagem nutricional evitará a deterioração e melhorará o estado nutricional desse grupo de pacientes.

Palavras-chave: ventilação mecânica não invasiva, terapia nutricional, insuficiência respiratória aguda.
- Centro de Investigación en Enfermedades Infecciosas, Instituto Nacional de Enfermedades Respiratorias, Ciudad de México, México.

*Correspondencia: Iván A. Osuna- Padilla. ivan.osuna@cieni.org.mx

\footnotetext{
2. Departamento de Nutrición, Hospital General de León, León, Guanajuato México.

3. Médico Especialista en Neumología, Instituto Nacional de Enfermedades Respiratorias, Ciudad de México, México.
}

\section{INTRODUCCIÓN}

El uso de ventilación mecánica no invasiva (VMNI) en el paciente con insuficiencia respiratoria aguda con el objetivo de evitar la intubación endotraqueal y otras complicaciones, se ha incrementado en los últimos años $^{(1)}$. Los pacientes tratados con dicha modalidad de ventilación presentan retos particulares en lo que respecta al abordaje nutricional. El principal problema es la prescripción rutinaria de ayuno durante las primeras 48 horas posteriores a la instauración de la VMNI prolongándose con frecuencia por más tiempo ${ }^{(2)}$. Esto se debe a que el inicio de la vía oral o nutrición enteral (NE) suele percibirse como poco seguro, al incrementar el riesgo de aspiración ${ }^{(3,4)}$ o provocar fugas de aire cuando no se utilizan mascarillas con adaptaciones para sonda de alimentación, mermando con ello la efectividad de la terapia ventilatoria. El ayuno impacta de forma negativa el estado nutricional ${ }^{(5)}$, provocando un mayor déficit de energía y proteína, lo cual se asocia a empeoramiento en la funcionalidad, pérdida de músculo y desarrollo de desnutrición ${ }^{(6)}$.

En pacientes con enfermedad pulmonar, la desnutrición empeora la función respiratoria, alterando los mecanismos nerviosos centrales que regulan la respiración y disminuyendo la capacidad para sostener niveles adecuados de ventilación por el deterioro de la musculatura respiratoria ${ }^{(7)}$.

La VMNI se define como todo modo de soporte ventilatorio que mejora la ventilación alveolar utilizando una máscara o dispositivo similar denominado interfaz ${ }^{(8)}$. Esta modalidad de ventilación fue conce- bida inicialmente para el tratamiento de los trastornos respiratorios durante el sueño, extendiéndose su uso al manejo de la insuficiencia respiratoria aguda a partir de la década de los $90^{(9)}$.

Los pacientes con insuficiencia respiratoria aguda son aquellos que han perdido la capacidad de generar un adecuado intercambio de oxígeno durante la respiración, debido a alteraciones pulmonares o en el sistema de bomba que lo hace funcionar. Es así como surge la administración de un volumen de aire a presión por la boca (presión positiva) como una alternativa para proveer al paciente de asistencia ventilatoria a fin de compensar su falla mecánica y disminuir las complicaciones ${ }^{(10)}$.

El uso de VMNI está indicado en ciertos tipos de insuficiencia respiratoria, específicamente en la hipercápnica o tipo II, como lo son las exacerbaciones de la Enfermedad Pulmonar Obstructiva Crónica (EPOC) o el edema agudo de pulmón ${ }^{(11)}$, así como en situaciones clínicas específicas como el retiro de la ventilación mecánica invasiva (VMI) en pacientes con EPOC o en pacientes con riesgo alto de falla al retiro. Una VMNI exitosa requiere una interfaz con un sello adecuado, ya sea mascarilla oronasal, facial completa o casco. Las contraindicaciones absolutas para su uso son la presencia de malformaciones faciales o trauma en conjunto con la obstrucción fija de la vía aérea, las contraindicaciones relativas el deterioro del estado de alerta (Escala de Coma de Glasgow $<8$ ) o un $\mathrm{pH}$ arterial $<7,15^{(12)}$.

La insuficiencia respiratoria aguda incrementa el riesgo de desnutrición, al presentarse una disminución en el consumo de alimentos en conjunto con un incremento en el gasto energético en reposo (GER), 
este último a consecuencia del estado inflamatorio y del incremento en el trabajo de los músculos respirato$\operatorname{rios}^{(13,14)}$. La desnutrición tiene un impacto directo en la inmunidad pulmonar, al disminuir la fagocitosis, la proliferación de linfocitos $\mathrm{T}$ y la secreción de IgA en dichos órganos ${ }^{(15)}$, presentándose un incremento en las tasas de infecciones, mayor estancia hospitalaria y mayor mortalidad ${ }^{(16)}$. No se cuenta con cifras de prevalencia de desnutrición en pacientes con VMNI, sin embargo, en Latinoamérica $40 \%$ - $60 \%$ de los pacientes hospitalizados la presentan ${ }^{(17)}$.

El objetivo de esta revisión narrativa es 1. analizar la literatura científica respecto al abordaje nutricional del paciente con insuficiencia respiratoria aguda y ventilación mecánica no invasiva, y 2. proponer un algoritmo de abordaje nutricional para prevenir el deterioro y mejorar el estado nutricional de pacientes con dicha condición clínica.

\section{CONTROVERSIAS EN EL ABORDAJE NUTRICIONAL EN PACIENTES CON VMNI E INSUFICIENCIA RESPIRATORIA AGUDA}

Con el objetivo de conocer las prácticas de abordaje nutricional del paciente con VMNI e insuficiencia respiratoria aguda, se realizó una búsqueda no sistemática de la literatura en PubMed e Hinari, utilizando las palabras clave (non invasive ventilation $O R$ noninvasive ventilation) AND (nutrition OR nutritional OR intake). Se incluyeron estudios primarios publicados en inglés, de enero de 2000 a mayo de 2019, realizados en adultos con lesión pulmonar aguda o insuficiencia respiratoria aguda. Se obtuvieron cuatro estudios ${ }^{(2,18-20)}$.

En el estudio de Reeves et al. se analizó el consumo dietético vía oral en 36 pacientes con VMNI, observando que más de $75 \%$ de los pacientes consumían menos de $80 \%$ de los requerimientos de energía y de proteína $^{(18)}$. Los autores sugieren el desarrollo de protocolos de abordaje nutricional para evitar las complicaciones asociadas a la desnutrición. Arnaout et al. estudiaron 90 pacientes que requirieron apoyo con VMNI durante más de 4 horas al día, observando un consumo menor a $1.000 \mathrm{kcal} /$ día en los primeros 5 días de esta modalidad ventilatoria. Los autores sugieren que se realicen estudios multicéntricos con el objetivo de evaluar el abordaje nutricional más óptimo ${ }^{(19)}$.

Kogo et al. analizaron de manera retrospectiva el riesgo de la nutrición enteral para pacientes con VMNI, en una cohorte de 107 pacientes que no podían alimentarse por vía oral. El $56 \%$ de estos pacientes recibieron
NE y $54 \%$ nutrición parenteral. En los individuos que recibieron NE se observó mayor incidencia de neumonía por aspiración ( $17 \% v s 4 \%, \mathrm{p}=0,044)$, de complicaciones de la vía aérea ( $53 \%$ vs $32 \%, \mathrm{p}=0,03)$, mayor duración de la VMNI (16 días vs 8 días, $\mathrm{p}=0,02$ ) y mayor estancia hospitalaria (48 días $v s 37$ días, $\mathrm{p}=<$ 0,001). El análisis multivariado mostró que la NE no se relacionó con la mortalidad intra hospitalaria. Los autores concluyen que la nutrición enteral debe ser considerada cuidadosamente en estos pacientes ${ }^{(20)}$.

Terzi et al. analizaron de manera retrospectiva el manejo nutricional inicial y los desenlaces clínicos en una cohorte conformada por 1.075 pacientes que recibieron VMNI durante al menos 2 días consecutivos. Se encontró que en los dos primeros días de VMNI 57,9\% no recibió nutrición, 2,6\% recibió NE, 6,9\% solo NP y $32,7 \%$ restante recibió únicamente vía oral. Los pacientes que recibieron NE en comparación con los que no recibieron nutrición (ayuno) presentaron una mayor mortalidad a los 28 días (HR 2,3 IC $95 \%$ 1,2 - 4,4, p $=0,01$ ), una mayor incidencia de infecciones (HR 2,2 IC $95 \% 1,1-4,5, \mathrm{p}=0,03)$ y menor número de días libres de ventilador (RR 0,70 IC $95 \% 0,5-0,9, p=$ $0,01)^{(2)}$. El ayuno o la subalimentación no se asociaron a mortalidad. Se concluyó que se requieren estudios adicionales para evaluar la necesidad, así como el mejor momento y la mejor ruta de terapia nutricional en esta población específica.

La capacidad deglutoria no se ve afectada por la VMNI, según lo observado en pacientes con $\mathrm{EPOC}^{(21)}$; por lo tanto, los pacientes pueden recibir alimentación vía oral, como lo reporta el estudio de Terzi et al. en $32,7 \%$ de los pacientes de su estudio. Es importante resaltar que en los pacientes que se alimentan por vía oral, el consumo de energía y proteínas puede ser subóptimo, ya que los pacientes suelen cursar con disnea por tiempos prolongados al retiro de la mascarilla, como lo reportan Reeves et al. y Arnaout et al. ${ }^{(18,19)}$.

Los resultados mencionados con anterioridad demuestran la necesidad de realizar estudios para elucidar el impacto del ayuno. Debería considerarse el impacto del déficit energético y proteico en pacientes con VMI, en quienes una menor provisión de proteínas en los primeros 3 días de estancia se asocia a una mayor estancia hospitalaria y un menor número de días libres de ventilador según lo reportado por Yeh et al. ${ }^{(22)}$ en pacientes en estado crítico. Otros autores han mostrado la importancia de maximizar los esfuerzos para lograr la ingesta o la administración de proteínas prescrita en UCI debido a que se ha observado una menor morta- 
lidad en aquellos pacientes que cumplen los requerimientos nutricionales ${ }^{(23,24)}$.

En conclusión, los pacientes que comienzan la VMNI para tratar la insuficiencia respiratoria aguda a menudo no pueden comer $y$, por lo tanto, permanecen en ayuno. Además, existe una paradoja en el abordaje nutricional de estos pacientes la cual consiste en considerar que el inicio inmediato de la vía oral y de NE como poco seguro, lo que contribuye a favorecer el ayuno en estos pacientes. Esto es por lo general, más frecuente en las primeras $48 \mathrm{~h}$ posteriores al inicio de la VMNI. Es importante resaltar que el ayuno no está exento de repercusiones en el estado de salud (Figura 1). Se necesitan más estudios de calidad para evaluar el impacto de la NE en los resultados, la mortalidad y la calidad de vida del paciente. En la práctica, la indicación de NE debe ser cuidadosamente considerada en estos pacientes.

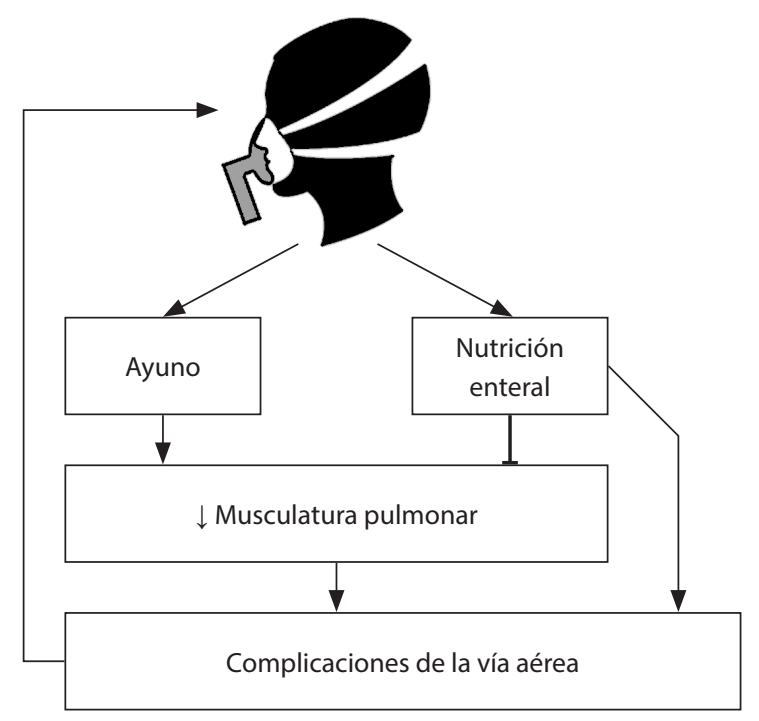

Figura 1. Implicaciones del ayuno y de la nutrición enteral en el paciente con ventilación mecánica no invasiva.

\section{LINEAMIENTOS DE SOPORTE NUTRICIONAL EN EL PACIENTE CON VENTILACIÓN MECÁNICA INVASIVA Y NO INVASIVA}

La implementación de los lineamientos de abordaje nutricional emitidos para pacientes con VMI puede ser de utilidad para prevenir el deterioro nutricional del paciente con VMNI. Algunas de las recomendaciones mencionadas en la literatura son:

- Evaluar la presencia de riesgo nutricional a través de herramientas de tamizaje, como la universal de detección de desnutrición (MUST) o la escala de riesgo nutricional (NRS-2002), que han mostrado tener una sensibilidad y especificidad superior al resto de las herramientas disponibles en la literatura, o bien identificar la presencia de desnutrición a través de la Valoración Global Subjetiva (VGS) ${ }^{(25)}$.

- La nutrición enteral (NE) es la vía de provisión de nutrientes más adecuada, es segura incluso en pacientes que reciben VMI en posición prono ${ }^{(26,27)}$. Se sugiere su inicio en las primeras 24 - 48 horas postintubación independiente del estado nutricional, al asociarse el inicio temprano a una menor mortali$\mathrm{dad}^{(28)}$, postergando su inicio en casos de inestabilidad hemodinámica que ameriten la prescripción de fármacos vasopresores ${ }^{(29,30)}$.

- La nutrición parenteral (NP) es la estrategia para utilizar en pacientes con alteraciones o disfunción del tracto gastrointestinal e incapacidad para su uso. En pacientes con riesgo nutricional en quienes se inició NE, se sugiere el inició de NP cuando han transcurrido de 3 a 5 días y no se ha logrado alcanzar $60 \%$ de las metas de energía y proteína ${ }^{(31)}$.

- Para la prescripción de cualquier modalidad de nutrición es de suma importancia conocer las necesidades nutricionales, las cuales son determinadas por el sexo, la edad, la composición corporal, la condición clínica y el esquema de medicamentos (debiendo considerar el aporte energético de soluciones glucosadas, propofol y citrato, entre otros). La calorimetría indirecta es considerada el estándar de oro para la determinación del GER en pacientes con $\mathrm{VMI}^{(32)}$; sin embargo, por su alto costo y poca disponibilidad, se suelen utilizar ecuaciones predictivas del GER, siendo las ecuaciones de Penn State University las que presentan mayor exactitud en la predicción ${ }^{(33)}$.

- Existe controversia respecto a la meta energética a alcanzar en pacientes con insuficiencia pulmonar aguda, ya que algunos autores han documentado que la provisión de $100 \%$ de la meta calórica puede tener resultados contraproducentes al inducir la supresión de la autofagia ${ }^{(34)}$, sugiriéndose la estrategia de hipoalimentación permisiva, la cual consiste en infundir $10-20 \mathrm{~mL} / \mathrm{h}$ de fórmula enteral ${ }^{(35)}$, más esta estrategia podría ser poco adecuada en aquellos pacientes desnutridos o en riesgo de estarlo, al exacerbar su condición ${ }^{(36)}$.

Los pacientes con insuficiencia pulmonar aguda, independientemente del tipo de ventilación mecánica que reciben, presentan un estado catabólico debido al estrés 
metabólico y a la respuesta inflamatoria sistémica ${ }^{(14)}$. En pacientes con VMI, la terapia nutricional óptima (NE o NP) ha mostrado mejorar los desenlaces clínicos, al disminuir la deuda calórica y proteica. Bajo esta premisa, el paciente con VMNI en riesgo nutricional o con desnutrición establecida debería recibir una intervención nutricional (NE o NP) de manera temprana en aquellos casos donde la vía oral no es factible, utilizando los lineamientos propuestos para maximizar los beneficios en el paciente con VMI.

\section{Recomendaciones dietéticas}

En aquellos pacientes que toleran el retiro de la VMNI por tiempo corto, se sugiere promover la alimentación vía oral. Ante la falta de consenso respecto a las pautas dietéticas específicas para estos pacientes, pueden implementarse las recomendaciones nutricionales emitidas para pacientes con EPOC, considerando los siguientes aspectos:

- Es importante asegurar que la dieta prescrita aporte las cantidades de energía y de proteína requeridas por el individuo ${ }^{(37,38)}$. No existen recomendaciones de energía y proteína específicas para pacientes con insuficiencia pulmonar aguda, se sugiere prescribir de $25-30 \mathrm{kcal} / \mathrm{kg}$ de peso corporal, aportando un rango de $1,2-2,0 \mathrm{~g} / \mathrm{kg}$ de proteína ${ }^{(39)}$.

- La prescripción de dieta líquida no confiere beneficios en materia de complicaciones, por el contrario, puede ser contraproducente considerando que el aporte de energía y proteína es subóptimo en la mayoría de los $\operatorname{casos}^{(40)}$.

- Algunas estrategias alimentarias pueden ayudar a incrementar el consumo de energía y proteína, entre ellas: 1. ofrecer comidas pequeñas de fácil masticación y alta densidad energética a lo largo del día; 2 . tratar de beber los líquidos al final del alimento para disminuir la saciedad temprana y 3. ofrecer alimentos a temperatura ambiente, ya que algunos pacientes pueden cursar con resequedad en la mucosa oral, lo cual incrementa la sensibilidad térmica ${ }^{(41)}$.

- La incorporación de un suplemento oral nutricional puede ser de gran utilidad en los pacientes que no logran cubrir entre $60 \%$ y $75 \%$ de sus requerimientos energéticos con la vía oral durante más de cinco días consecutivos, o si presentan desnutrición. Su incorporación a la terapia nutricional permite promover un balance energético positivo, además de documentarse mejorías en la masa muscular y en la funcionalidad ${ }^{(37,42,43)}$. No se sugiere el uso rutinario de fórmulas especializadas para patología pulmonar, ya que no han demostrado beneficio clínico ${ }^{(34)}$, ni de fórmulas enriquecidas con omega 3 y antioxidantes, las cuales no han mostrado mejorar la oxigenación y la mecánica ventilatoria según lo reportado por un metaanálisis de 10 ensayos clínicos ${ }^{(44)}$.

\section{PROPUESTA DE ALGORITMO DE ABORDAJE NUTRICIONAL}

La implementación de algoritmos de abordaje nutricional permite mejorar las prácticas en materia de soporte nutricional y la provisión de nutrientes ${ }^{(45)}$. Para la elaboración del algoritmo propuesto en la Figura 2, se consultaron además de la literatura ya mencionada, las guías y documentos de práctica segura de la Sociedad Alemana de Nutrición Clínica (DGEM) ${ }^{(46)}$, las guías de nutrición clínica en la unidad de cuidados intensivos publicadas por la Sociedad Europea de Nutrición Clínica y Metabolismo (ESPEN) en 2019 ${ }^{(47)}$ y las guías de soporte metabólico y nutricional de la Sociedad Americana de Nutrición Parenteral y Enteral (ASPEN) publicadas en 2016.

Las guías de nutrición clínica en medicina crítica publicadas en 2019 por la DGEM sugieren el inicio de NE en pacientes con VMNI sin alteración en el estado de conciencia o sin trastornos de la deglución, utilizando preferentemente la interfaz de casco para disminuir el riesgo de aspiración. Las guías de práctica clínica y documentos de consenso publicados por ESPEN y ASPEN no mencionan las características del abordaje nutricional en el paciente con VMNI.

Basados en la evidencia mencionada en esta revisión narrativa recomendamos el siguiente algoritmo. En pacientes con desnutrición o con riesgo nutricional, que se espera que sean incapaces de cubrir sus requerimientos de energía y proteína con alimentación por la vía oral, se sugiere prescribir NE en las primeras 24 - 48 horas de estancia hospitalaria. En aquellos pacientes con riesgo nutricional en quienes no es posible instaurar un acceso enteral, la prescripción de NP de forma inmediata resulta esencial para disminuir el déficit energético y proteico. Independiente del riesgo nutricional, la NP suplementaria debería iniciarse utilizando la vía de acceso más adecuada para el paciente (central o periférica) al tercer día de no lograr un aporte de $60 \%$ de los requerimientos energéticos por vía oral o enteral. Por supuesto, se debe considerar la evolución, estado de salud y pronóstico del paciente ${ }^{(48)}$. La terapia nutricional tardía, después del día 8 según lo estipulado 


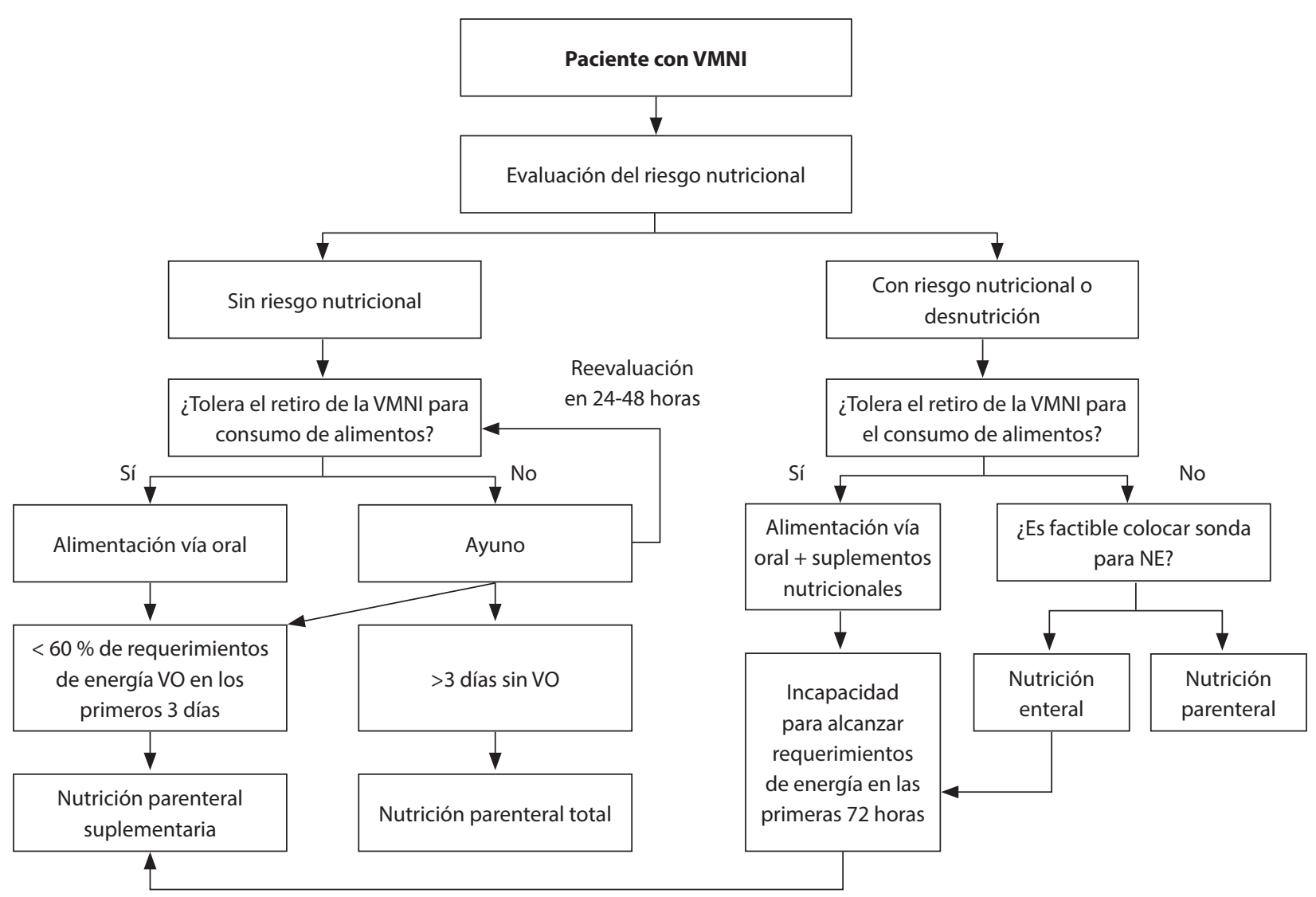

Figura 2. Algoritmo de intervención nutricional en el paciente con ventilación mecánica no invasiva. La intervención nutricional en el paciente con VMNI debe prescribirse considerando el riesgo nutricional, promoviendo la alimentación vía oral y el uso de suplementos nutricionales orales en aquellos pacientes que toleran el retiro de la VMNI, e iniciando de forma temprana la nutrición enteral y parenteral para disminuir la deuda calórica y proteica.

por ASPEN ${ }^{(35)}$ se asocia a mayor déficit energético y mayores complicaciones. Las nuevas terapias disponibles, como el oxígeno nasal de alto flujo, deben ser consideradas, ya que pueden permitir una alimentación oral más apropiada ${ }^{(3)}$.

\section{CONCLUSIÓN}

En la actualidad no existe consenso para el abordaje nutricional de pacientes con VMNI. Sin embargo, considerando las asociaciones entre el déficit de energía y proteína y los desenlaces clínicos reportados en el paciente hospitalizado, resulta fundamental la implementación de algoritmos de abordaje nutricional como el propuesto que permitan la prescripción de una terapia nutricional óptima. Esta terapia incluye el uso de suplementos orales nutricionales, nutrición enteral o parenteral en los casos que se amerite. El objetivo es evitar el ayuno prolongado. Se requieren estudios de intervención que permitan elucidar la terapia nutricional más óptima en el paciente con insuficiencia respiratoria y ventilación mecánica no invasiva, así como la validación del algoritmo de abordaje propuesto.

\section{Financiamiento}

El presente estudio no tuvo financiación.

\section{Conflicto de intereses}

Sin conflictos de interés por declarar.

\section{Declaración de autoría}

Todos los autores participaron en la concepción y realización del trabajo que ha dado como resultado el artí- 
culo en cuestión. Todos los autores revisaron el artículo y validaron su versión final.

\section{Referencias bibliográficas}

1. De Jong A, Hernandez G, Chiumello D. Is there still a place for noninvasive ventilation in acute hypoxemic respiratory failure? Intensive Care Med. 2018; 44(2): 2248-50.

2. Terzi N, Darmon M, Reignier J, Ruckly S, Garrouste-Orgeas $\mathrm{M}$, Lautrette $\mathrm{A}$, et al. Initial nutritional management during noninvasive ventilation and outcomes: a retrospective cohort study. Crit Care. 2017; 21(1):293.

3. Singer $\mathrm{P}$, Rattanachaiwong $\mathrm{S}$. To eat or to breathe? The answer is both! Nutritional management during noninvasive ventilation. Crit Care. 2018; 22(1): 27.

4. Gay PC. Complications of noninvasive ventilation in acute care. Respir Care. 2009;54(2):246-57.

5. Pichard C, Oshima T, Berger MM. Energy deficit is clinically relevant for critically ill patients: yes. Intensive Care Med. 2015; 41(2):335-38.

6. Fetterplace K, Beach LJ, MacIsaac C, Presneill J, Edbrooke L, Parry SM, et al. Associations between nutritional energy delivery, bioimpedance spectroscopy and functional outcomes in survivors of critical illness. J Hum Nutr Diet. 2019. Abril 29. Abril Dec;32(6):702-712. doi: 10.1111/jhn.12659.

7. Ferrari-Baliviera E, Pierdominici S, Sarcinelli L. Effects of the nutritional status on the respiratory system. Minerva Anestesiol. 1989;55(11):443-50.

8. British Thoracic Society Standards of Care Committee. Non-invasive ventilation in acute respiratory failure. Thorax. 2002;57(2):192-211.

9. Storre JH, Callegari JC. Matching mode and settings to the patient: an introduction. En: Simonds AK. ERS Practical Handbook of Noninvasive Ventilation. London: European Respiratory Society; 2015. p:18-25.

10. Thille AW, Frat JP. Noninvasive ventilation as acute therapy. Curr Opin Crit Care. 2018;24(6):519-24.

11. Rochwerg B, Brochard L, Elliott MW, Hess D, Hill NS, Nava $S$, et al. Official ERS/ATS clinical practice guidelines: noninvasive ventilation for acute respiratory failure. Eur Respir J. 2017;50(2):pii: 1602426.

12. Davidson AC, Banham S, Elliott M, Kennedy D, Gelder C, Glossop A, et al. BTS/ICS guideline for the ventilatory management of acute hypercapnic respiratory failure in adults. Thorax. 2016;71(Suppl 2):ii1-35.

13. Rochester DF, Braun NMT, Laine S. Diaphragmatic energy expenditure in chronic respiratory failure: The effect of assisted ventilation with body respirators. Am J Med. 1977;63(2):223-32.

14. Loi M, Wang J, Ong C, Lee JH. Nutritional support of critically ill adults and children with acute respiratory distress syndrome: A clinical review. Clin Nutr ESPEN. 2017;19:1-8.
15. Ghignone M, Quintin L. Malnutrition and respiratory function. Int Anesthesiol Clin. 1986;24(1):65-74.

16. Lew $\mathrm{CCH}$, Yandell R, Fraser RJL, Chua AP, Chong MFF, Miller M. Association Between Malnutrition and Clinical Outcomes in the Intensive Care Unit: A Systematic Review. JPEN J Parenter Enteral Nutr. 2017;41(5):744-58.

17. Correia MITD, Perman MI, Waitzberg DL. Hospital malnutrition in Latin America: A systematic review. Clin Nutr. 2017;36(4):958-67.

18. Reeves A, White H, Sosnowski K, Tran K, Jones M, Palmer M. Energy and protein intakes of hospitalised patients with acute respiratory failure receiving non-invasive ventilation. Clin Nutr. 2014;33(6):1068-73.

19. Arnaout M, Marincamp A, Reffiena M, Debue A-S, Le Bras $\mathrm{M}$, Boulila C, et al. Systematic evaluation of intakes in patients receiving non invasive ventilation. the starve study. Intensive Care Med Exp. 2015;3(Suppl 1): A827.

20. Kogo M, Nagata K, Morimoto T, Ito J, Sato Y, Teraoka S, et al. Enteral Nutrition Is a Risk Factor for Airway Complications in Subjects Undergoing Noninvasive Ventilation for Acute Respiratory Failure. Respir Care. 2017;62(4):459-67.

21. Terzi N, Normand H, Dumanowski E, Ramakers M, Seguin A, Daubin C, et al. Noninvasive ventilation and breathingswallowing interplay in chronic obstructive pulmonary disease*. Crit Care Med. 2014;42(3):565-73.

22. Yeh DD, Fuentes E, Quraishi SA, Lee J, Kaafarani HMA, Fagenholz P, et al. Early Protein Inadequacy Is Associated With Longer Intensive Care Unit Stay and Fewer Ventilator-Free Days: A Retrospective Analysis of Patients With Prolonged Surgical Intensive Care Unit Stay. JPEN Parenter Enteral Nutr. 2018;42(1):212-8.

23. Nicolo M, Heyland DK, Chittams J, Sammarco T, Compher C. Clinical Outcomes Related to Protein Delivery in a Critically Ill Population: A Multicenter, Multinational Observation Study. JPEN Parenter Enteral Nutr. 2016;40(1):45-51.

24. Compher C, Chittams J, Sammarco T, Nicolo M, Heyland DK. Greater Protein and Energy Intake May Be Associated with Improved Mortality in Higher Risk Critically Ill Patients: A Multicenter, Multinational Observational Study. Crit Care Med. 2017;45(2):156-63.

25. van Bokhorst-de van der Schueren MAE, Guaitoli PR, Jansma EP, de Vet HCW. Nutrition screening tools: does one size fit all? A systematic review of screening tools for the hospital setting. Clin Nutr. 2014;33(1):39-58.

26. Allen K, Hoffman L. Enteral Nutrition in the Mechanically Ventilated Patient. Nutr Clin Pract. 2019. En prensa

27. Saez de la Fuente I, Saez de la Fuente J, Quintana Estelles MD, Garcia Gigorro R, Terceros Almanza LJ, Sanchez Izquierdo JA, et al. Enteral Nutrition in Patients Receiving Mechanical Ventilation in a Prone Position. JPEN Parenter Enteral Nutr. 2016;40(2):250-5.

28. Reignier J, Darmon M, Sonneville R, Borel A-L, GarrousteOrgeas M, Ruckly S, et al. Impact of early nutrition and feeding route on outcomes of mechanically ventilated patients with 
shock: a post hoc marginal structural model study. Intensive Care Med. 2015;41(5):875-86.

29. Bruns BR, Kozar RA. Feeding the Postoperative Patient on Vasopressor Support: Feeding and Pressor Support. Nutr Clin Pract. 2016;31(1):14-7.

30. Reintam Blaser A, Starkopf J, Alhazzani W, Berger MM, Casaer MP, Deane AM, et al. Early enteral nutrition in critically ill patients: ESICM clinical practice guidelines. Intensive Care Med. 2017;43(3):380-98.

31. Worthington P, Balint J, Bechtold M, Bingham A, Chan L-N, Durfee $S$, et al. When Is Parenteral Nutrition Appropriate? JPEN J Parenter Enteral Nutr. 2017; 41(3):324-77.

32. Rattanachaiwong $S$, Singer P. Should we calculate or measure energy expenditure? practical aspects in the ICU. Nutrition. 2018;55-56:71-5.

33. Tatucu-Babet OA, Ridley EJ, Tierney AC. Prevalence of Underprescription or Overprescription of Energy Needs in Critically Ill Mechanically Ventilated Adults as Determined by Indirect Calorimetry: A Systematic Literature Review. JPEN J Parenter Enteral Nutr. 2016;40(2):212-25.

34. Van Dyck L, Casaer MP, Gunst J. Autophagy and Its Implications Against Early Full Nutrition Support in Critical Illness. Nutr Clin Pract. 2018;33(3):339-47.

35. McClave SA, Taylor BE, Martindale RG, Warren MM, Johnson DR, Braunschweig C, et al. Guidelines for the Provision and Assessment of Nutrition Support Therapy in the Adult Critically Ill Patient: Society of Critical Care Medicine (SCCM) and American Society for Parenteral and Enteral Nutrition (A.S.P.E.N.). JPEN J Parenter Enteral Nutr. 2016;40(2):159-211.

36. Stuever MF, Kidner RF, Chae FE, Evans DC. Full Nutrition or Not?. Nutr Clin Pract. 2018;33(3):333-8.

37. Schols AM, Ferreira IM, Franssen FM, Gosker HR, Janssens $\mathrm{W}$, Muscaritoli M, et al. Nutritional assessment and therapy in COPD: a European Respiratory Society statement. Eur Respir J. 2014;44(6):1504-20.

38. National Health and Medical Research Council. Noninvasive ventilation guidelines for adult patients with acute respiratory failure. [Internet]. Australia: National Health and Medical
Research Council, 2014 [Fecha de consulta: mayo 6 de 2019]. Disponible en: https://www.clinicalguidelines.gov.au/portal/2514/noninvasive-ventilation-guidelines-adult-patientsacute-respiratory-failure

39. McClave SA, DiBaise JK, Mullin GE, Martindale RG. ACG Clinical Guideline: Nutrition Therapy in the Adult Hospitalized Patient. Am J Gastroenterol. 2016; 111(3):315-34.

40. Rattray M, Desbrow B, Roberts S. Comparing nutritional requirements, provision and intakes among patients prescribed therapeutic diets in hospital: An observational study. Nutrition. 2017;39-40:50-6.

41. Fernandes AC, Bezerra OM de PA. Nutrition therapy for chronic obstructive pulmonary disease and related nutritional complications. J Bras Pneumol. 2006; 32(5):461-71.

42. Ferreira IM, Brooks D, White J, Goldstein R. Nutritional supplementation for stable chronic obstructive pulmonary disease. Cochrane Database Syst Rev. 2012;12:CD000998.

43. Collins PF, Stratton RJ, Elia M. Nutritional support in chronic obstructive pulmonary disease: a systematic review and metaanalysis. Am J Clin Nutr. 2012; 95(6):1385-95.

44. Dushianthan A, Cusack R, Burgess VA, Grocott MP, Calder PC. Immunonutrition for acute respiratory distress syndrome (ARDS) in adults. Cochrane Database of Systematic Reviews. 2019. 1: CD012041.

45. Kiss CM, Byham-Gray L, Denmark R, Loetscher R, Brody RA. The impact of implementation of a nutrition support algorithm on nutrition care outcomes in an intensive care unit. Nutr Clin Pract. 2012;27(6):793-801.

46. Elke G, Hartl WH, Kreymann KG, Adolph M, Felbinger TW, Graf T, et al. Clinical Nutrition in Critical Care Medicine - Guideline of the German Society of Clinical Nutrition (DGEM). Clin Nutr ESPEN. 2019. Mayo 9. En prensa.

47. Singer P, Blaser AR, Berger MM, Alhazzani W, Calder PC, Casaer MP, et al. ESPEN guideline on clinical nutrition in the intensive care unit. Clin Nutr. 2019;38(1):48-79.

48. Russell MK, Wischmeyer PE. Supplemental Parenteral Nutrition: Review of the Literature and Current Nutrition Guidelines. Nutr Clin Pract. 2018;33(3):359-69. 